\title{
Efficacy of alcohol/sugar aqueous biphasic system on partition of bovine serum albumin
}

\author{
Yin Hui Chow ${ }^{1}$, Alagan Sahlini ${ }^{1}$, Hui-Suan $\mathrm{Ng}^{2^{*}}$ and John Chi-Wei Lan ${ }^{3^{*}}$
}

\begin{abstract}
The efficacy of alcohol/sugar aqueous biphasic (ABS) system on protein extraction was investigated. A model protein, bovine serum albumin (BSA), was adopted to evaluate the effects of types and concentration of phase-forming components, protein concentration, and system pH on the protein partition efficiency. The 1-propanol/maltose ABS exhibited an overall better partition efficiency of BSA to the alcohol-rich top phase. A maximum partition coefficient $(K)$ of $20.01 \pm 0.05$ and recovery yield $(Y)$ of $95.42 \% \pm 0.01 \%$ of BSA were achieved with $35 \%(w / w) 1-p r o p a n o l / 22 \%$ $(\mathrm{w} / \mathrm{w})$ maltose $\mathrm{ABS}$ at $\mathrm{pH} 5.0$ for $10 \%(\mathrm{w} / \mathrm{w}) \mathrm{BSA}$ load. The $K$ and $Y$ of BSA in 1-propanol/maltose ABS was slightly improved with the addition of $3 \%(\mathrm{w} / \mathrm{w}$ ) of ionic liquid, 1-butyl-3-methylimidazolium bromide ([Bmim] Br) as the adjuvant that could provide protein stabilizing effect. The Fourier Transform Infrared Spectrum (FTIR) analysis revealed that the protein structure remained unaltered upon the separation process.
\end{abstract}

Keywords: Aqueous biphasic system, Separation, Protein partition, Alcohol, Carbohydrate

\section{Introduction}

Aqueous biphasic system (ABS) with high water content has emerged as an alternative cost-effective approach for protein separation and purification. ABS is formed by mixing two immiscible phase-forming solutions at a critical composition (Ng et al. 2011). The difference in the physicochemical properties and the interactions between the biomolecules and the phase-forming molecules results in differential partitioning and separation of the target biomolecules in the ABS. ABS offers several process advantages, such as low interfacial tension, short phase separation time, and low toxicity. Polymer/polymer ABS, polymer/salt ABS, and alcohol/salt ABS have been widely applied in protein extraction (Ng et al. 2011). Nevertheless, the industrial-scale applications of polymer-based ABS are limited because of the high viscosity

\footnotetext{
*Correspondence: GrraceNg@ucsiuniversity.edu.my; lanchiwei@saturn.yzu. edu.tw

2 Faculty of Applied Sciences, UCSI University, No. 1, Jalan Menara Gading, UCSI Heights, Cheras, 56000 Kuala Lumpur, Malaysia

${ }^{3}$ Biorefinery and Bioprocess Engineering Laboratory, Department of Chemical Engineering and Materials Science, Yuan Ze University, Chungli, Taoyuan 320, Taiwan

Full list of author information is available at the end of the article
}

of polymer used and the high cost associated with the recycling of phase-forming components (Ng et al. 2012). Moreover, the use of high charge density salts may cause environmental issues and denaturation of targeted protein due to its high ionic strength or alkalinity.

The substitution of conventional salts with carbohydrates could potentially create a more biocompatible separation environment for the biomolecules (de Brito Cardoso et al. 2013). Carbohydrates are polyhydroxy aldehydes or ketones that encompass a broad range of organic compounds. It can be divided into two large groups: simple sugars (i.e. monosaccharides) and compound sugars (i.e. oligosaccharides and polysaccharides). Sugars are electrically neutral, non-toxic, biodegradable, and highly hydrophilic (Sadeghi et al. 2016). Sugars possess several hydroxyl groups with dual donor/acceptor characteristics that can participate in hydrogen bonding, thereby exerting sugaring-out effect (i.e., also known as soluting-out effect) inherently (de Brito Cardoso et al. 2013). Rising attention has been given to the application of carbohydrates as a sugaring-out agent to form polymer/sugar ABS (Sadeghi et al. 2016), acetonitrile/sugar ABS (de Brito Cardoso et al. 2013), ionic liquid (IL)/ sugar ABS (Quental et al. 2018), and alcohol/sugar ABS 
(Ebrahimi and Sadeghi 2018). The viscosity of alcohol is lower when compared to polymers and ILs, allowing more rapid phase separation and efficient mass transfer of biomolecules from one phase to another (Wang et al. 2010). Considering these advantages, alcohol/sugar ABS could potentially serve as a low-cost and sustainable extraction platform for the recovery and separation of biomolecules. Although the phase composition and properties of alcohol/sugar ABS have been characterized (Ebrahimi and Sadeghi 2018), the separation efficiency of the alcohol/sugar ABS and the stability of the biomolecules after extraction remain superficial to date.

This study aims to investigate the partition efficiency of protein in alcohol/sugar ABS composed of short-chain aliphatic alcohols (1-propanol and 2-propanol) and different sugars (glucose, sucrose, and maltose). Carbohydrates, such as glucose, sucrose, and maltose, are low-cost sugars that can be easily derived from natural sources and are widely used in the food industries. Longer chain alcohol (e.g. butanol) has higher protein destabilizing power than the shorter and branched one (e.g. methanol, ethanol, and 1-propanol) and thus was not investigated in this study (Miyawaki and Tatsuno 2011). Serum albumin is the most abundant protein in vertebrates' body fluid. It has numerous vital physiological functions. Bovine serum albumin (BSA), which has $76 \%$ structural similarity with human serum albumin (HSA), has been extensively applied as the model protein in various extraction and purification studies (Pereira et al. 2015). Hence, BSA was employed as the model protein to evaluate the efficacy of alcohol/sugar ABS on the partition of protein in this study.

ILs are often known as designer solvents due to their flexibility and tunability. These greener solvents are generally non-flammable, chemically stable, and possess high dissolving capacity and good extractability. Besides its application as phase-forming components, recent studies have shown that smaller amounts $(\leq 5 \%(\mathrm{w} / \mathrm{w}))$ of ILs can be added as adjuvants into polymer/salt ABS (Neves et al. 2019; Aziz et al. 2017) and alcohol/salt (Ran et al. 2019) to enhance the partition efficiency of biomolecules to one of the phases. In this study, the effects of the addition of neutral salts and ILs as adjuvants on the partition efficiency of BSA in alcohol/sugar ABS were examined. The stability of BSA upon the ABS separation process was also assessed with Fourier Transform Infrared Spectrum (FTIR) analysis.

\section{Materials and methods Materials}

Lyophilized BSA (essentially globulin and fatty acid free, $\geq 99 \%$ pure) was purchased from Sigma-Aldrich (St. Louis, MO, USA). Ethanol absolute $99.8 \%\left(\mathrm{C}_{2} \mathrm{H}_{5} \mathrm{OH}\right)$, 1-propanol $\left(\mathrm{C}_{3} \mathrm{H}_{8} \mathrm{O}\right)$ absolute $99.5 \%$, and 2-propanol absolute $99.5 \%$ were obtained from VWR Chemicals (Fontenay-Sous-Bois, France). D-(+)-glucose ( $\geq 99.0 \%)$, sucrose $(\geq 98.0 \%)$, and $\mathrm{D}-(+)$-maltose monohydrate $(\geq 99.0 \%)$ were obtained from Merck (Darmstadt, Germany). Pierce BCA protein assay kit was sourced from Thermo Scientific (Rockford, United States of America).

\section{Determination of binodal curves}

The binodal curves were constructed according to the turbidimetric titration method using different concentrations of alcohol (i.e. 1-propanol and 2-propanol) and sugar (i.e. glucose, maltose, and sucrose) (Johansson et al. 2011). A mixture containing known concentrations of alcohol and sugar stock solution was weighed and titrated dropwise with an appropriate amount of ultrapure water until a single-phase solution was formed. The resultant mixture was centrifuged at $4000 \mathrm{~g}$ for $10 \mathrm{~min}$ to ensure a single-phase system was formed. The final weight of the system was measured to determine the amount of ultrapure water added.

\section{Protein partitioning in alcohol/sugar ABS}

The protein partitioning experiment was performed at room temperature $\left(25^{\circ} \mathrm{C}\right)$. ABS with a final mass of $5.0 \mathrm{~g}$ was prepared in a $15-\mathrm{mL}$ centrifuge tube by mixing appropriate amounts of $100 \%(\mathrm{w} / \mathrm{w})$ alcohol (i.e. 1-propanol and 2-propanol), $60 \%(\mathrm{w} / \mathrm{w})$ stock solution of sugar (i.e. glucose, maltose, and sucrose), ultrapure water, and $5 \%(\mathrm{w} / \mathrm{w})$ of $20 \mathrm{mg} / \mathrm{mL}$ of BSA protein solution unless otherwise stated (Ng et al. 2018). The final protein concentration in $\mathrm{ABS}$ was approximately equivalent to the BSA concentration (ca. $1 \mathrm{mg} / \mathrm{mL}$ ) tested in other works and thus could provide the possibility for comparison of protein partition efficiency in various types of ABS. Subsequently, the mixture was centrifuged at $4000 \mathrm{~g}$ for $10 \mathrm{~min}$ to ensure total phase separation was attained. The volume of each phase was recorded. Sample was collected separately from each phase to quantify the BSA concentration. All ABS partitioning experiments were performed in triplicate.

\section{Determination of protein content using bicinchoninic acid (BCA) assay}

The concentration of BSA in each phase was determined using the bicinchoninic acid (BCA) assay (Ng et al. 2018). To each of the $25 \mu \mathrm{L}$ of phase sample solution, $200 \mu \mathrm{L}$ of working reagent was added. The resultant mixture was mixed thoroughly for $30 \mathrm{~s}$ and incubated at $37{ }^{\circ} \mathrm{C}$ for $30 \mathrm{~min}$. Next, the absorbance of the resultant mixture was measured at $562 \mathrm{~nm}$ against the blank ABS phase sample solution which was prepared in parallel to eliminate any possible interferences. The calibration curve was 
constructed using BSA standard solutions diluted to the working range of $25-2000 \mu \mathrm{g} / \mathrm{mL}$.

\section{Fourier Transform Infrared Spectrum (FTIR) analysis}

The Fourier-Transform Infrared Spectrometer (FTIR) analysis was conducted to examine the protein conformation and stability of BSA before and after the ABS extraction (Pereira et al. 2015). The FT-IR spectra $\left(4 \mathrm{~cm}^{-1}\right.$ resolution, 16 scans) were recorded using the Perkin Elmer Spectrum 100 FT-IR spectrometer (BioTek, Winooski, VT, USA) in the wavelength ranging from 450 to $4000 \mathrm{~cm}^{-1}$ to locate the main functional group.

\section{Determination of protein partition efficiency}

The volume ratio $\left(V_{R}\right)$ was expressed as the ratio of the volume of the top phase $\left(V_{T}\right)$ to the volume of the bottom phase $\left(V_{B}\right)$ :

$$
V_{R}=\frac{V_{T}}{V_{B}} .
$$

The partition coefficient $(K)$ of BSA was calculated as the ratio of the concentration of BSA at the top phase $\left(C_{T}\right)$ to the concentration of BSA at the bottom phase $\left(C_{B}\right)$ (Eq. 2):

$$
K=\frac{C_{T}}{C_{B}}
$$

The recovery yield $(Y)$ was used as a parameter to evaluate the percentage of the amount of BSA partitioned between the top phase and the total mixture. The $Y$ was calculated as a function of $V_{R}$ and $K$ according to (Eq. 3) (Ng et al. 2019):

$$
Y=\frac{100}{1+\frac{1}{V_{R} \cdot K}} .
$$

\section{Results and discussion}

\section{Binodal curves of sugaring-out assisted ABS}

Figure 1a and $\mathrm{b}$ depicts the binodal curves of 1-propanol/sugar $A B S$ and 2-propanol/sugar ABS, respectively. The biphasic region of 1-propanol/sugar ABS was larger than that of 2-propanol/sugar ABS. This trend indicates that 1-propanol exhibits greater phaseforming ability compared to 2-propanol. According to previous report, the octanol/water partition coefficient $\left(K_{o w}\right)$ value of 1-propanol is 1.78 and the $K_{o w}$ of 2-propanol is 1.12 (Ebrahimi and Sadeghi 2018). The higher $K_{o w}$ value of 1-propanol isomer suggests that it has higher hydrophobicity and lower solubility in water and polar substances. Furthermore, the aqueous 1-propanol solution has a higher surface tension compared to the aqueous 2-propanol solution, thereby facilitating
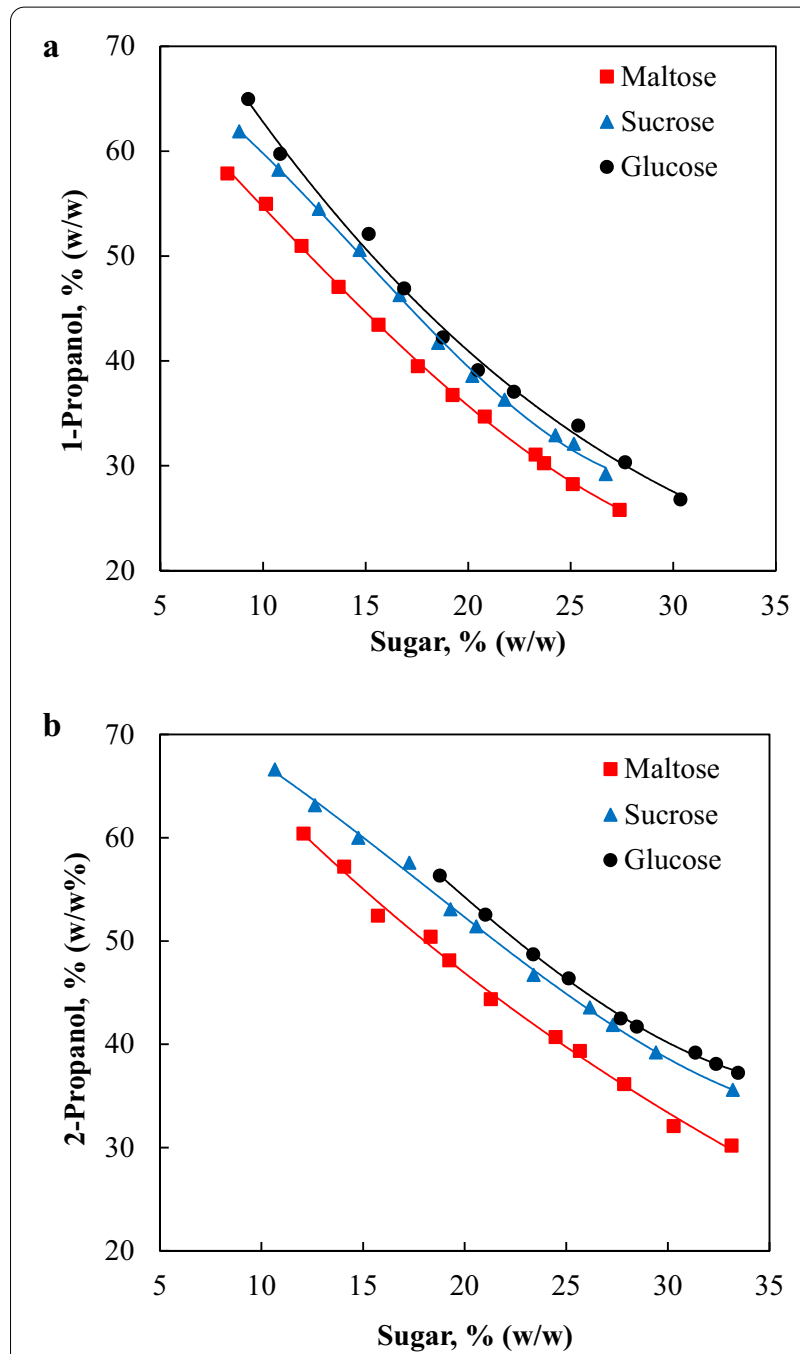

Fig. 1 Binodal curves of alcohol/sugar ABS. a Binodal curves of 1-propanol/sugar ABS and $\mathbf{b}$ Binodal curves of 2-propanol/sugar ABS. The solid lines of the binodal curves were obtained by connecting the sufficient points experimentally

more effective phase separation (Erfani et al. 2019). Thus, a lower concentration of 1-propanol is sufficient to form ABS with sugar. Ethanol was also investigated as a potential phase-forming component for the formation of alcohol/sugar ABS. Several systems constituted with various concentrations of ethanol and sugar were examined. However, no phase formation was observed. This could be due to the relatively close hydrophilicity degree between the ethanol and the sugars investigated (Ebrahimi and Sadeghi 2018). Moreover, precipitation was observed in the mixture which contained a high concentration of ethanol and a low concentration of sugar. Thus, ethanol and sugars are not suitable candidates to form stable alcohol/sugar ABS. 
Figure 1a shows that the binodal curve of 1-propanol/ maltose ABS was closer to the axes compared to the binodal curves of 1-propanol/glucose ABS and 1-propanol/sucrose ABS. A similar trend was observed for 2-propanol/sugar ABS (Fig. 1b), indicating that maltose has higher phase-forming ability compared to sucrose and glucose. The trend was in agreement with the sugaring-out ability and hydrophilicity of the sugars investigated. It has been reported that the ability of these sugars in sugaring-out ionic liquids (Ferreira et al. 2016), polymers (Sadeghi et al. 2016), and alcohols (Ebrahimi and Sadeghi 2018) for the formation of ABS increased in the order of glucose $<$ sucrose $<$ maltose. As shown in Fig. 2, the disaccharide maltose possesses a higher number of equatorial hydroxyl groups compared to sucrose and glucose, thereby leading to a higher affinity of maltose for water and stronger sugaring-out ability (Freire et al. 2011; Ebrahimi and Sadeghi 2016). Thus, the amount of maltose required to form ABS with 1-propanol and 2-propanol was lower compared to sucrose and glucose as shown in Fig. 1.

\section{Effect of types and concentrations of phase-forming components on the partition efficiency of BSA}

The partitioning behaviour of BSA in 1-propanol/sugar $\mathrm{ABS}$ and 2-propanol/sugar ABS was investigated to evaluate the effect of various types and concentrations of phase-forming components on the partition efficiency of BSA. The $K$ and recovery yield $(Y)$ of BSA in the alcoholrich top phase are shown in Table 1 . The investigated systems were chosen based on their relative position in the biphasic region which gave a $V_{R}$ of 1.0 at equilibrium.

Results in Table 1 show that the 1-propanol/sugar ABS demonstrated better partition efficiency of BSA than 2-propanol/sugar ABSs. This could be attributed to the relatively higher hydrophobicity of 1-propanol compared to 2-propanol that promotes the partition of BSA towards the alcohol-rich top phase of 1-propanol/sugar ABS (Ng et al. 2018). Furthermore, 1-propanol/sugar
ABSs with maltose-rich bottom phase gave an overall better protein partition efficiency compared to 1-propanol/sucrose ABS and 1-propanol/glucose ABS. A similar trend was observed in the 2-propanol/sugar ABS. The effect of types of sugar on the protein partition efficiency could be explained by the sugaring-out ability of sugars and their hydration extensions (Ebrahimi and Sadeghi 2018). The intensity of the sugaring-out effect depends on the differential position and the number of hydroxyl groups on the pyranose ring. Equatorial hydroxyl groups possess greater hydration potential than the axial ones as the former could interact with water molecules and form long-lived hydration structures ( $\mathrm{Ng}$ et al. 2012; Sadeghi et al. 2016). For glucose, its C-2, C-3, and C-4 hydroxyl groups are all in the equatorial positions (Fig. 2). Maltose and sucrose possess the same number of hydroxyl group. Maltose is composed of two glucose monomers, whereas sucrose is composed of a glucose monomer and a fructose monomer. As such, maltose which consists of two six-membered pyranose rings is more easily hydrated than sucrose (Freire et al. 2011). In other words, maltose which has a higher number of equatorial hydroxyl groups could exert a stronger sugaring-out effect than sucrose and glucose, thereby improving the transfer of BSA to the alcohol-rich top phase.

Based on Table 1, the optimal condition for BSA extraction was observed in 35\% (w/w) 1-propanol/22\% $(\mathrm{w} / \mathrm{w})$ maltose ABS, which had a maximum $K$ of $6.03 \pm 0.17$ and $Y$ of $87.84 \% \pm 0.61 \%$. These results indicate that the partition efficiency of BSA is better at lower concentrations of 1-propanol and maltose. As the alcohol-rich top phase is more hydrophobic compared to the sugar-rich bottom phase, the hydrophobic interactions between the BSA and 1-propanol-rich phase are probably the main driving force for the preferential partition of BSA to the alcoholrich top phase (Ng et al. 2018).

In general, the increase in alcohol and sugar concentrations were unfavourable for the partition of BSA in 1-propanol/sugar ABSs and 2-propanol/sugar ABSs.
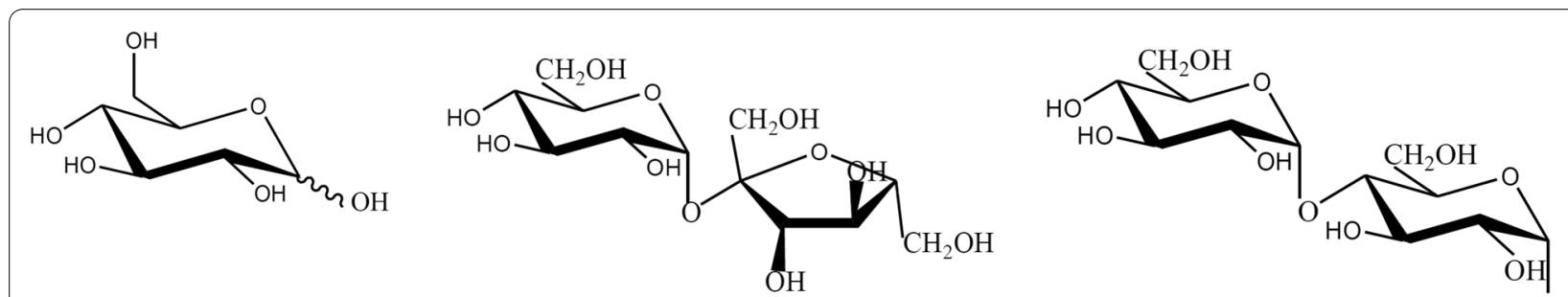

Glucose

Sucrose

Maltose

Fig. 2 Molecular structure of sugars used to form the alcohol/sugar ABS 
Table 1 Effect of types and concentration of phase-forming components on the partition efficiency of BSA with propanol/sugar ABS

\begin{tabular}{|c|c|c|c|c|}
\hline Types of ABS & $\begin{array}{l}\text { Concentration of sugar, } \% \\
(w / w)\end{array}$ & $\begin{array}{l}\text { Concentration of alcohol, \% } \\
(w / w)\end{array}$ & $K$ & Recovery yield, $\%$ \\
\hline \multirow[t]{5}{*}{ 1-Propanol/Maltose } & 22 & 35 & $6.03 \pm 0.17$ & $87.84 \pm 0.61$ \\
\hline & 23 & 37 & $4.40 \pm 0.43$ & $81.70 \pm 1.88$ \\
\hline & 24 & 38 & $2.57 \pm 0.46$ & $72.58 \pm 3.58$ \\
\hline & 25 & 39 & $1.74 \pm 0.14$ & $64.39 \pm 1.81$ \\
\hline & 26 & 39 & $0.90 \pm 0.25$ & $49.40 \pm 4.79$ \\
\hline \multirow[t]{5}{*}{ 1-Propanol/Sucrose } & 26 & 33 & $1.17 \pm 0.07$ & $63.61 \pm 1.48$ \\
\hline & 27 & 34 & $1.26 \pm 0.11$ & $56.71 \pm 2.11$ \\
\hline & 28 & 35 & $1.36 \pm 0.15$ & $62.86 \pm 2.64$ \\
\hline & 29 & 36 & $0.93 \pm 0.10$ & $48.21 \pm 2.72$ \\
\hline & 30 & 37 & $0.72 \pm 0.08$ & $42.33 \pm 3.32$ \\
\hline \multirow[t]{5}{*}{ 1-Propanol/Glucose } & 24 & 36 & $1.14 \pm 0.08$ & $50.42 \pm 2.10$ \\
\hline & 25 & 38 & $1.36 \pm 0.05$ & $58.11 \pm 1.48$ \\
\hline & 26 & 39 & $1.01 \pm 0.08$ & $50.76 \pm 2.58$ \\
\hline & 27 & 40 & $0.59 \pm 0.02$ & $38.04 \pm 0.71$ \\
\hline & 28 & 41 & $0.38 \pm 0.09$ & $30.75 \pm 4.91$ \\
\hline \multirow[t]{5}{*}{ 2-Propanol/Maltose } & 31 & 32 & $2.41 \pm 0.39$ & $70.51 \pm 3.35$ \\
\hline & 32 & 33 & $1.19 \pm 0.19$ & $54.19 \pm 3.88$ \\
\hline & 33 & 34 & $0.85 \pm 0.03$ & $42.10 \pm 0.92$ \\
\hline & 34 & 35 & $0.76 \pm 0.14$ & $43.06 \pm 4.63$ \\
\hline & 35 & 36 & $0.33 \pm 0.03$ & $23.38 \pm 1.66$ \\
\hline \multirow[t]{5}{*}{ 2-Propanol/Sucrose } & 33 & 38 & $1.01 \pm 0.03$ & $48.13 \pm 0.84$ \\
\hline & 34 & 39 & $0.75 \pm 0.02$ & $43.02 \pm 0.60$ \\
\hline & 35 & 40 & $0.74 \pm 0.04$ & $42.46 \pm 1.19$ \\
\hline & 36 & 41 & $0.22 \pm 0.05$ & $17.71 \pm 3.15$ \\
\hline & 37 & 41.5 & $0.08 \pm 0.03$ & $7.42 \pm 2.90$ \\
\hline \multirow[t]{5}{*}{ 2-Propanol/Glucose } & 31 & 39 & $0.82 \pm 0.07$ & $44.95 \pm 2.00$ \\
\hline & 32 & 40 & $0.59 \pm 0.00$ & $39.84 \pm 0.10$ \\
\hline & 33 & 41 & $0.35 \pm 0.05$ & $27.38 \pm 2.70$ \\
\hline & 34 & 42 & $0.32 \pm 0.04$ & $25.82 \pm 2.51$ \\
\hline & 35 & 43 & $0.48 \pm 0.02$ & $34.33 \pm 1.10$ \\
\hline
\end{tabular}

Alcohol/sugar ABSs comprised of different phase-forming component concentration were selected for investigation based on their relative position in the biphasic region which gave a $V_{R}$ of 1.0 at equilibrium

Further increase of alcohol and sugar concentrations reduced the $K$ to below unity $(K<1)$ and decreased the $Y$ to below $50 \%$ for all the investigated alcohol/ sugar ABSs. This decrease in partition efficiency could be attributed to the gradual dehydration of both aqueous phases as the phase-forming components' concentration increases, but to a stronger extent in the alcohol-rich top phase (Ebrahimi and Sadeghi 2018). This dehydration effect resulted in insufficient free water molecules to solubilize the protein in the alcoholrich top phase and exclusion of protein to the bottom phase, thereby resulting in the decrease of protein partition efficiency (Ooi et al. 2009). Therefore, 35\% (w/w) 1-propanol/22\% (w/w) maltose ABS which has the highest protein partition efficiency was selected for further studies.

\section{Effect of BSA amount added to the ABS on the partition efficiency of BSA}

The amount of BSA added into the ABS was varied at a range of $5-25 \%(\mathrm{w} / \mathrm{w})$ to evaluate the partition efficiency of BSA in the $35 \%(\mathrm{w} / \mathrm{w}) 1$-propanol $/ 22 \%(\mathrm{w} / \mathrm{w})$ maltose ABS (Fig. 3). When the amount of protein added to the system was increased from $5 \%(\mathrm{w} / \mathrm{w})$ to $10 \%(\mathrm{w} / \mathrm{w})$, the partition coefficient increased from $6.27 \pm 0.16$ to $10.21 \pm 0.03$ and the recovery yield increased from $87.12 \% \pm 1.55 \%$ to $91.27 \% \pm 0.02 \%$. It was observed that the increase in protein amount from $5 \%(\mathrm{w} / \mathrm{w})$ to $25 \%$ 


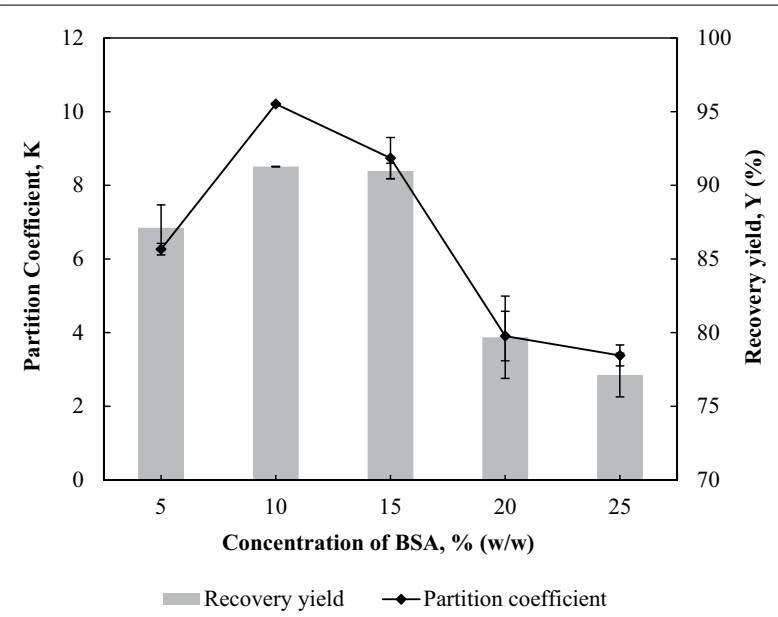

Fig. 3 Effect of amount of BSA added to the ABS on the partition efficiency of BSA. The amount of BSA was varied at a range of $5-25 \%$ $(\mathrm{W} / \mathrm{w})$ to evaluate the effect of protein concentration on the $K$ and $Y$ of BSA in the $35 \%(\mathrm{w} / \mathrm{w}) 1$-propanol/22\% $(\mathrm{w} / \mathrm{w})$ maltose ABS. The results are expressed as mean \pm standard deviation of triplicate readings

$(\mathrm{w} / \mathrm{w})$ resulted in a decrease of $V_{R}$ by $8.7 \%$. With the decrease in $V_{R}$, the free volume which is available in the top phase to accommodate a higher amount of protein is greatly reduced (Ooi et al. 2009). Hence, the protein partition efficiency decreased to a minimum $K$ of $3.38 \pm 0.28$ and $Y$ of $77.12 \% \pm 1.49 \%$ when the protein amount was increased to $25 \%(\mathrm{w} / \mathrm{w})$. The increase in the amount of BSA beyond $25 \%(\mathrm{w} / \mathrm{w})$ is not feasible due to the increase in the tendency of protein precipitation at the interface as a result of phase saturation ( $\mathrm{Ng}$ et al. 2014). Thus, $10 \%$ $(\mathrm{w} / \mathrm{w})$ of BSA solution which exhibited the highest protein partition efficiency was selected to further investigate the effect of $\mathrm{pH}$ on the partitioning behaviour of BSA in the 1-propanol/maltose ABS.

\section{Effect of $\mathrm{pH}$ on the partition efficiency of BSA}

The $\mathrm{pH}$ of an $\mathrm{ABS}$ can be adjusted to steer and enhance the partition of protein to the targeted phase. In this study, the $\mathrm{pH}$ of the $35 \%(\mathrm{w} / \mathrm{w}) 1$-propanol $/ 22 \%(\mathrm{w} / \mathrm{w})$ maltose ABS containing $10 \%(\mathrm{w} / \mathrm{w})$ BSA was varied between $\mathrm{pH} 3.0$ and $\mathrm{pH} 8.0$ to investigate the effect of $\mathrm{pH}$ on the partition efficiency of BSA. This $\mathrm{pH}$ range was selected by considering the isoelectric point $(\mathrm{pI}=4.8)$ of BSA and its relative stability at $\mathrm{pH} 5.0-8.0$ (Chow et al. 2015). The phase system was mixed well while adjusting the $\mathrm{pH}$ with the addition of $1.0 \mathrm{M}$ of sulphuric acid or $1.0 \mathrm{M}$ sodium hydroxide. The change in the volume of both phases was negligible with the increase in $\mathrm{pH}$.

As shown in Fig. 4, the $\mathrm{pH}$ exerted a significant impact on the protein partition efficiency. When the $\mathrm{pH}$ was increased from $\mathrm{pH} 3.0$ to $\mathrm{pH}$ 5.0, the partition efficiency

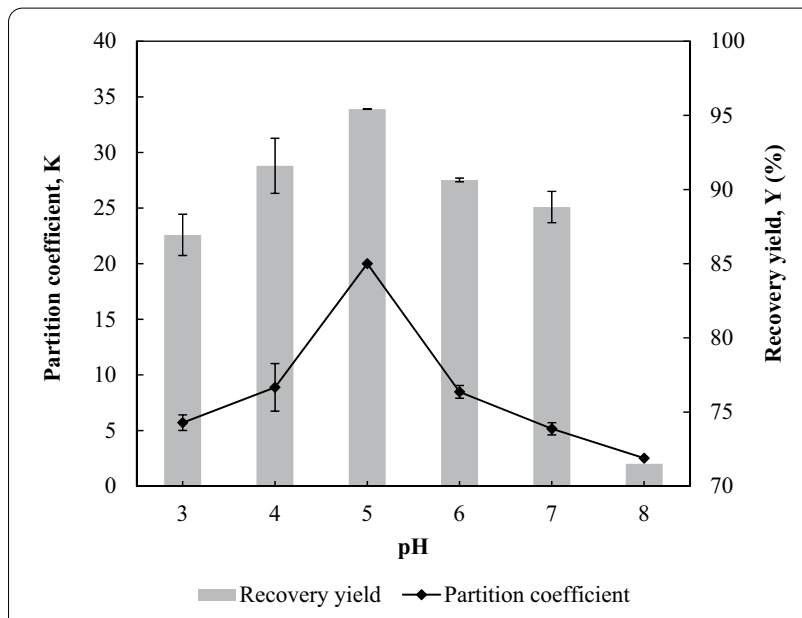

Fig. 4 Effect of $\mathrm{pH}$ on the partition efficiency of BSA. The $\mathrm{pH}$ of the $35 \%(\mathrm{w} / \mathrm{w}) 1$-propanol/22\% (w/w) maltose ABS with 10\% (w/w) BSA was varied between $\mathrm{pH} 3.0$ and $\mathrm{pH} 8.0$ to investigate the effect of $\mathrm{pH}$ on the $K$ and $Y$ of BSA. The results are expressed as mean \pm standard deviation of triplicate readings

of BSA increased remarkably from $K$ of $5.71 \pm 0.7$ and $Y$ of $86.94 \% \pm 1.39 \%$ at $\mathrm{pH} 3.0$ to maximum $K$ of $20.01 \pm 0.05$ and $Y$ of $95.42 \% \pm 0.01 \%$ at $\mathrm{pH}$ 5.0. When the $\mathrm{pH}$ was increased to $\mathrm{pH}$ 5.0, approaching the $\mathrm{pI}$ of BSA, the net surface charge of BSA was close to zero (Chow et al. 2015). Thus, the hydrophobic interaction between the BSA and 1-propanol molecules was intensified at this $\mathrm{pH}$, facilitating the partition of more BSA to the alcohol-rich top phase (Ng et al. 2018). Thereafter, the protein partition efficiency decreased significantly when the $\mathrm{pH}$ was raised to $\mathrm{pH}$ 8.0. At $\mathrm{pH} 8.0$, the $K$ and $Y$ of BSA dropped to a minimum of $2.52 \pm 0.27$ and $71.50 \% \pm 2.17 \%$, respectively. No protein precipitation was observed for all the investigated $\mathrm{pH}$ values. As proteins prone to denature at high $\mathrm{pH}$ values, operating the ABS above $\mathrm{pH} 8.0$ would not be favourable for effective protein recovery (Chow et al. 2015).

\section{Effect of types of adjuvants on the partition efficiency of BSA}

The effects of the addition of neutral salts (sodium chloride $(\mathrm{NaCl})$ and potassium chloride $(\mathrm{KCl})$ ) or ILs (1-butyl3-methylimidazolium tetrafluoroborate, $\left[\mathrm{Bmim}^{-} \mathrm{BF}_{4}\right.$; 1-ethyl-3-methylimidazolium tetrafluoroborate, [Emim] $\mathrm{BF}_{4}$; 1-butyl-3-methylimidazolium bromide, $[\mathrm{Bmim}] \mathrm{Br}$; and 1-ethyl-3methylimidazolium bromide, [Emim] Br) on the $K$ and $Y$ of BSA in the 35\% (w/w) 1-propanol $/ 22 \%$ (w/w) maltose ABS at pH 5.0 were investigated (Fig. 5). The concentration of adjuvant added into the ABS was fixed at $1 \%(\mathrm{w} / \mathrm{w})$ and kept at a minimal value to ensure negligible change in phase composition and $V_{R}$. 


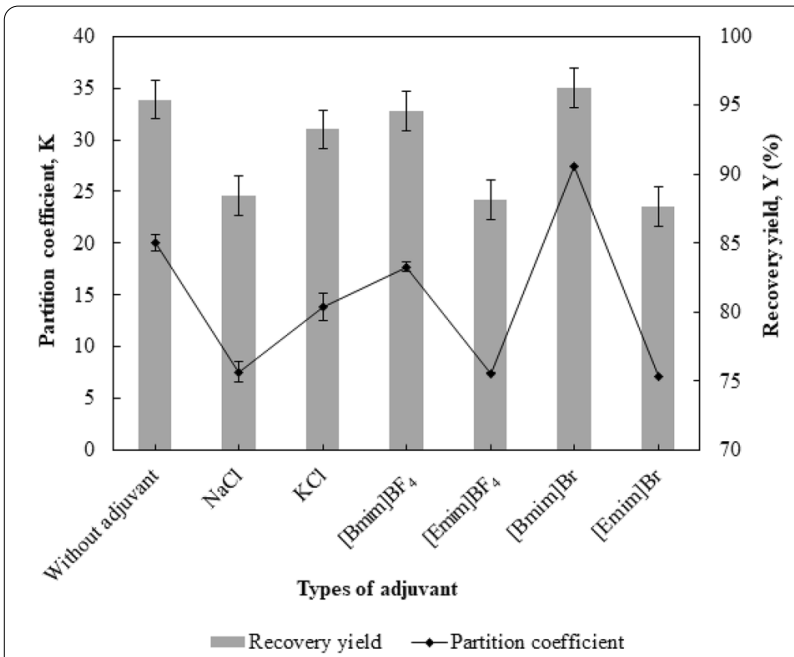

Fig. 5 Effect of types of adjuvants on the partition efficiency of BSA. Neutral salts, such as $\mathrm{NaCl}$ and $\mathrm{KCl}$, and ILs, such as [Bmim] BF ${ }_{4}$ [ [Emim] $\mathrm{BF}_{4},[\mathrm{Bmim}] \mathrm{Br}$ and $[\mathrm{Emim}] \mathrm{Br}$, were added at a fixed concentration of $1 \%(\mathrm{w} / \mathrm{w})$ to the $35 \%(\mathrm{w} / \mathrm{w}) 1$-propanol/22\% (w/w) maltose ABS at $\mathrm{pH}$ 5.0 as adjuvants to evaluate the effect of types of adjuvant on the $K$ and $Y$ of BSA. The results are expressed as mean \pm standard deviation of triplicate readings

According to the Hofmeister series, the saltingout effect is more pronounced for $\mathrm{KCl}$ than $\mathrm{NaCl}$ (Wan et al. 2018). Thus, higher $K(13.86 \pm 1.03)$ and $Y$ $(93.26 \% \pm 0.47 \%)$ were obtained in the $35 \%(\mathrm{w} / \mathrm{w}) 1$-propanol $/ 22 \%(\mathrm{w} / \mathrm{w})$ maltose ABS which was added with $\mathrm{KCl}$ compared to $K(7.57 \pm 0.78)$ and $Y(88.48 \% \pm 1.34 \%)$ obtained with the addition of $\mathrm{NaCl}$. Nevertheless, the $K$ and $Y$ values attained with the addition of these univalent neutral salts were lower than that without the addition of adjuvants, indicating that the presence of $\mathrm{KCl}$ and $\mathrm{NaCl}$ has a negative impact on the partition efficiency of BSA in the alcohol/sugar ABS.

Among the ILs investigated, only [Bmim] Br exhibited a positive impact on the protein partition efficiency. When compared to the alcohol/sugar ABS without adjuvants, the addition of $[\mathrm{Bmim}] \mathrm{BF}_{4}$ showed insignificant changes in the protein partition efficiency, while the addition of [Emim] $\mathrm{BF}_{4}$ and [Emim] Br reduced the $K$ and $Y$ significantly by more than $60 \%$ and $8 \%$, respectively. When the effect of IL's cation on the protein partition efficiency was compared, the $K$ and $Y$ values of BSA for ILs which share the same type of anion increase in the following orders: $[$ Emim $] \mathrm{BF}_{4}<[\mathrm{Bmim}] \mathrm{BF}_{4}$ and $[\mathrm{Emim}] \mathrm{Br}<[\mathrm{Bmim}] \mathrm{Br}$. This phenomenon could be attributed to the alkyl chain length of IL's cation. Longer alkyl chain length increases the hydrophobicity of ILs and enhances the molecular interaction between BSA and IL, thereby promoting the partition of the BSA in the top phase containing 1-propanol and IL (Ran et al. 2019). For the same alkyl chain length,

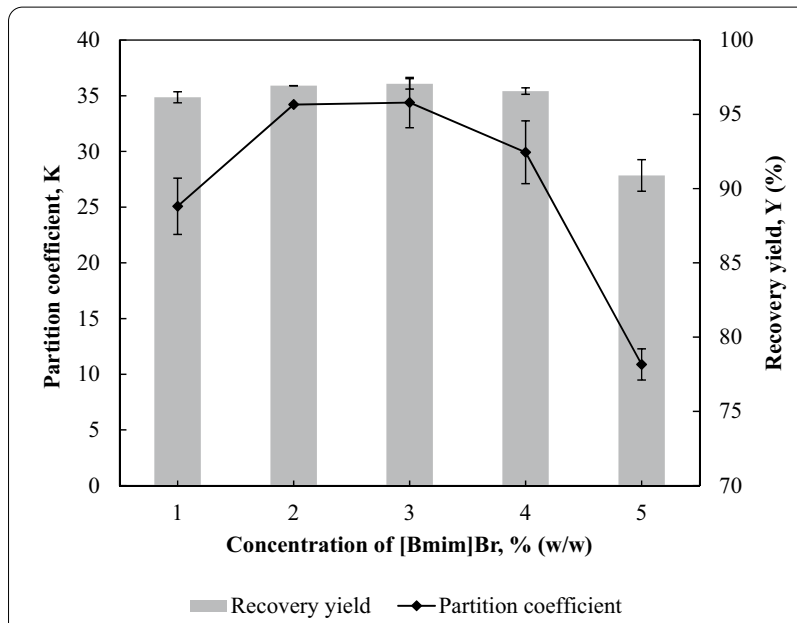

Fig. 6 Effect of concentration of adjuvant [Bmim] Br on the partition efficiency of BSA. The effect of [Bmim]Br's concentration ranging from $1.0 \%(\mathrm{w} / \mathrm{w})$ to $5.0 \%(\mathrm{w} / \mathrm{w})$ on the $K$ and $Y$ of BSA was evaluated. The results are expressed as mean \pm standard deviation of triplicate readings

[Bmim] Br exhibited higher $K$ and $Y$ values compared to $[\mathrm{Bmim}] \mathrm{BF}_{4}$. This variation in the protein partition efficiency is in accordance with the chaotropic order of the $\mathrm{IL}$ anions $\left(\mathrm{BF}_{4}^{-}>\mathrm{Br}^{-}\right)$, whereby the chaotropic $\mathrm{BF}_{4}^{-}$which has a higher tendency in unfolding the protein and destabilizing the hydrophobic aggregates will increase the dissolution of protein in the sugar-rich bottom phase (Ran et al. 2019). Thus, the addition of tetrafluoroborate-based IL decreased the partition efficiency of protein in the alcohol/sugar ABS. Comparing to the ABS without adjuvant ( $K$ of $20.01 \pm 0.05$ and $Y$ of $95.42 \% \pm 0.01 \%$ ), a slight increase in $K(27.42 \pm 0.02)$ and $Y(96.27 \% \pm 0.10 \%)$ were observed with the addition of $[\mathrm{Bmim}] \mathrm{Br}$. To further study the effect of addition of IL, [Bmim] Br was chosen to evaluate the effect of the concentration of IL on the partition efficiency of protein in the subsequent experiment.

\section{Effect of concentration of adjuvant [Bmim]Br on the partition efficiency of BSA}

The effect of [Bmim]Br's concentration on the partitioning behaviour of BSA was evaluated within the range of $1.0-5.0 \%(\mathrm{w} / \mathrm{w})$, and the results are presented in Fig. 6. When the concentration of $[\mathrm{Bmim}] \mathrm{Br}$ was increased to $3.0 \%(\mathrm{w} / \mathrm{w})$, there was a slight increase in $Y(97.05 \% \pm 0.35 \%)$ by $1.63 \%$ and a marked increase in $K(34.39 \pm 2.26)$ by $72 \%$ compared to the system without adjuvant. This slight increase in protein partition efficiency indicates that the addition of IL could serve to enhance the affinity of the 1-propanol-rich phase to the protein (Ran et al. 2019). The protein partition efficiency decreased thereafter to $K$ of $10.08 \pm 1.4$ and $Y$ of 
$90.88 \% \pm 1.06 \%$ at $5 \%(\mathrm{w} / \mathrm{w})[\mathrm{Bmim}] \mathrm{Br}$. This decrease in protein partition efficiency is probably caused by the change in protein stability at a high concentration of IL (Hadzir et al. 2016).

\section{Stability of BSA in 1-propanol/maltose ABS}

The FTIR spectra of pure BSA and BSA partitioned in the alcohol-rich top phase of the 35\% (w/w) 1-propanol/22\% $(\mathrm{w} / \mathrm{w})$ maltose ABS at $\mathrm{pH} 5$ with and without the addition of $3 \%(\mathrm{w} / \mathrm{w})$ [Bmim] Br IL are illustrated in Fig. 7. FTIR spectra could provide useful information for determining the protein secondary structure based on the presence of energy absorption bands of specific functional groups. Proteins are made up of many amino acids that are joined to one another by amide bonds. The $\mathrm{C}=\mathrm{O}$ bonds of the amide are involved in hydrogen bonding between different elements of the protein secondary structure. The amide I band which falls between 1600 and 17,000 $\mathrm{cm}^{-1}$ is associated with the $\mathrm{C}=\mathrm{O}$ stretching and ring stretching vibrations of the amide functional group (Pei et al. 2009). As the most sensitive spectral region to the secondary structure of protein, this characteristic amide
I absorption band is often used as a structural probe to determine the structural properties of protein.

In Fig. 7, the amide I absorption band of the pure BSA falls between 1600 and $17,000 \mathrm{~cm}^{-1}$. For the spectra of BSA separated to the top phase of 1-propanol/maltose ABS with and without IL, the amide I absorption band was readily identifiable in the same region (i.e., 1600$17,000 \mathrm{~cm}^{-1}$ ) without any shift caused by the presence of the ABS's phase-forming components and the added adjuvant. These observations demonstrated that the spatial structure of the BSA could be conserved in the phase solution of the 1-propanol/maltose ABS.

\section{Conclusion}

This work examined the partition efficiency of BSA in ABSs composed of various alcohols (i.e., 1-propanol and 2-propanol) and sugars (i.e., glucose, sucrose, and maltose). The types and concentration of phase-forming components, protein concentration, and $\mathrm{pH}$ have shown significant effect on the partition efficiency of protein. At low concentrations of alcohol and sugar, the BSA partitioned preferentially to the alcohol-rich top phase of the alcohol/sugar ABS. Maximum protein

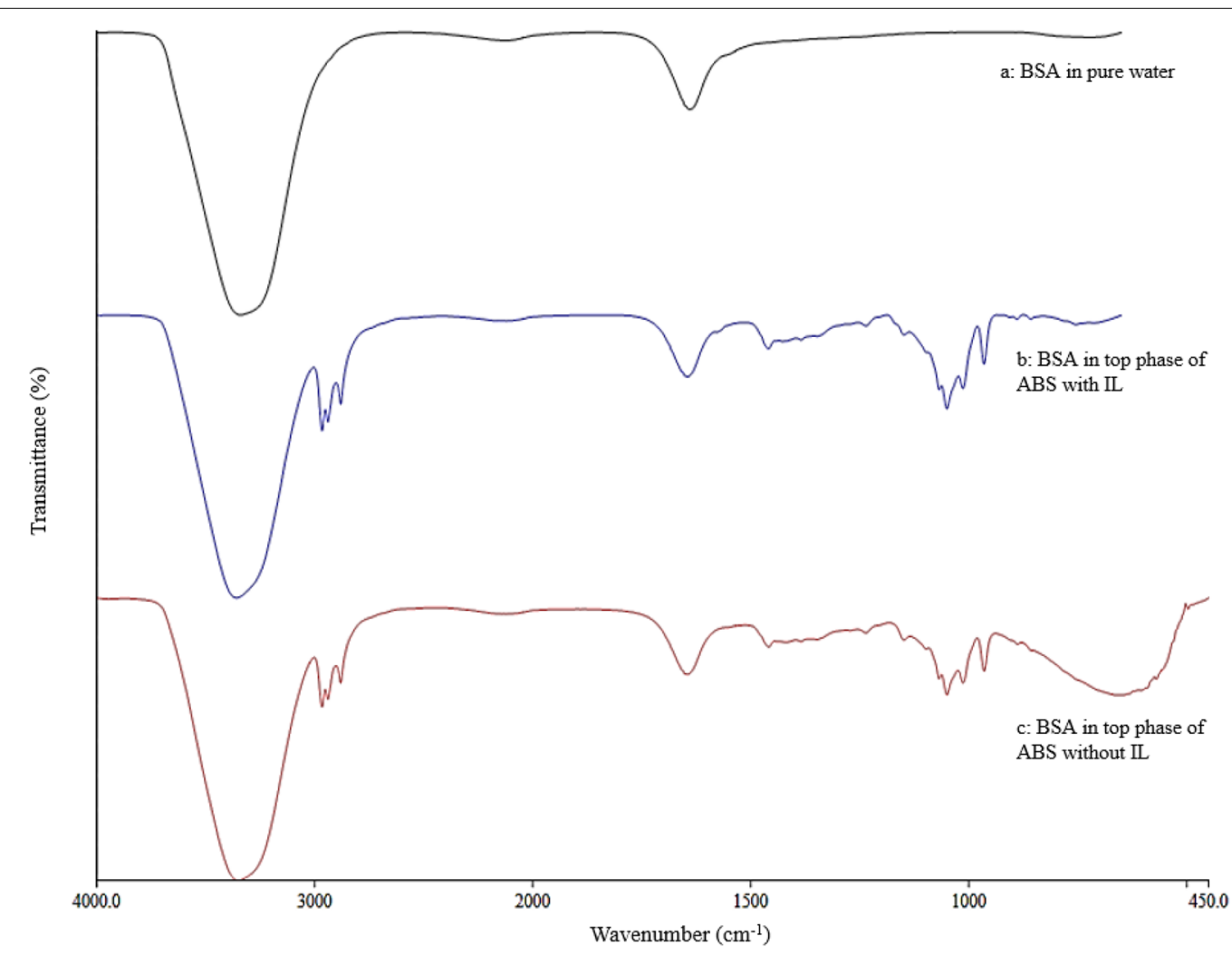

Fig. 7 FTIR spectra of BSA in various solution. a BSA in pure water; b BSA partitioned in the alcohol-rich top phase of the 35\% (w/W) 1-propanol/22\% (w/w) maltose ABS at pH 5 and added with 3\% (w/W) [Bmim] Br; c BSA partitioned in the alcohol-rich top phase of the 35\% (w/w) 1-propanol/22\% (w/w) maltose ABS at pH 5 
partition efficiency with $K$ of $20.01 \pm 0.05$ and $Y$ of $95.42 \% \pm 0.01 \%$ could be achieved in the $35 \% 1$-propanol/22\% (w/w) maltose ABS at $\mathrm{pH} 5.0$ which contained $10 \%(\mathrm{w} / \mathrm{w})$ BSA. The FTIR analysis showed that the BSA structure was conserved in the 1-propanol/ maltose ABS. A slight improvement in the partition efficiency of BSA was observed with the addition of $3 \%$ $(\mathrm{w} / \mathrm{w})$ of $[\mathrm{Bmim}] \mathrm{Br}$ as adjuvant into the ABS. However, considering the additional processing cost incurred for large-scale implementation, the addition of IL is not recommended. Comparing to the expensive IL/sugar $\mathrm{ABS}$, highly viscous polymer/sugar ABS, and the use of inorganic salt in polymer/salt ABS which could lead to corrosion of equipment and environmental pollution, the alcohol/sugar ABS could serve as a green and costeffective alternative for the separation and purification protein.

\begin{abstract}
Abbreviations
ABS: Aqueous biphasic system; (Bmim)BF $\mathrm{BF}_{4}$ 1-Butyl-3-methylimidazolium tetrafluoroborate; (Bmim)Br: 1-Butyl-3-methylimidazolium bromide; BCA: Bicinchoninic acid; BSA: Bovine serum albumin; C: Carbon; (Emim)BF 4 : 1-Ethyl3-methylimidazolium tetrafluoroborate; (Emim)Br: 1-Ethyl-3methylimidazolium bromide; FTIR: Fourier Transform Infrared Spectrum; HSA: Human serum albumin; IL: Ionic liquids; K: Partition coefficient; KCl: Potassium chloride; $\mathrm{NaCl}$ : Sodium chloride; O: Oxygen; $V_{R}:$ Volume ratio; Y: Yield.
\end{abstract}

\section{Acknowledgements}

The authors thank Taylor's University, Malaysia; UCSI University, Malaysia and Biorefinery and Bioprocess Engineering Laboratory, Yuan Ze University, Taiwan for the lab facilities.

\section{Authors' contributions}

YHC contributed to data curation, methodology, validation, and writing original draft. AS contributed to data curation and writing-original draft. HSN was involved in conceptualization, funding acquisition, and writing - review and editing. JCWL was involved in conceptualization, funding acquisition, supervision, and writing — review and editing. All authors read and approved the final manuscript.

\section{Funding}

This work was financially supported by Fundamental Research Grant Scheme (Grant Number: FRGS/1/2020/TKO/UCSI/02/6) from Ministry of Higher Education, Malaysia, and Ministry of Science and Technology Taiwan (Grant Number: MOST108-2622-E-155-013-CC2).

\section{Availability of data and materials}

All the datasets obtained in this study are included in this article.

\section{Ethics approval and consent to participate}

Not applicable.

\section{Consent for publication}

Not applicable.

\section{Competing interests}

The authors declare that they have no competing interests.

\footnotetext{
Author details

${ }^{1}$ School of Computer Science and Engineering, Faculty of Information and Technology, Taylor's University Lakeside Campus, 47500 Subang Jaya, Selangor, Malaysia. ${ }^{2}$ Faculty of Applied Sciences, UCSI University, No. 1, Jalan Menara Gading, UCSI Heights, Cheras, 56000 Kuala Lumpur, Malaysia.

${ }^{3}$ Biorefinery and Bioprocess Engineering Laboratory, Department of Chemical
}

Engineering and Materials Science, Yuan Ze University, Chungli, Taoyuan 320, Taiwan.

Received: 22 October 2020 Accepted: 4 January 2021

Published online: 21 January 2021

\section{References}

Aziz NFHA, Abbasiliasi S, Ng HS, Phapugrangkul P, Bakar MHA, Tam YJ, Tan JS (2017) Purification of $\beta$-mannanase derived from Bacillus subtilis ATCC 11774 using ionic liquid as adjuvant in aqueous two-phase system. J Chromatogr B 1055:104-112. https://doi.org/10.1016/j.jchro mb.2017.04.029

Chow YH, Yap YJ, Tan CP, Anuar MS, Tejo BA, Show PL, Ariff AB, Ng E-P, Ling TC (2015) Characterization of bovine serum albumin partitioning behaviors in polymer-salt aqueous two-phase systems. J Biosci Bioeng 120(1):8590. https://doi.org/10.1016/j.jbiosc.2014.11.021

de Brito Cardoso G, Mourão T, Pereira FM, Freire MG, Fricks AT, Soares CMF, Lima ÁS (2013) Aqueous two-phase systems based on acetonitrile and carbohydrates and their application to the extraction of vanillin. Sep Purif Technol 104:106-113. https://doi.org/10.1016/j.seppur.2012.11.001

Ebrahimi N, Sadeghi R (2016) Osmotic properties of carbohydrate aqueous solutions. Flu Pha Equi 417:171-180. https://doi.org/10.1016/j.fluid .2016 .02 .030

Ebrahimi N, Sadeghi R (2018) Propanol-Sugar aqueous biphasic systems as a suitable platform for biomolecules extraction. J Chromatogr A 1581-1582:156-167. https://doi.org/10.1016/j.chroma.2018.11.007

Erfani A, Khosharay S, Aichele CP (2019) Surface tension and interfacial compositions of binary glycerol/alcohol mixtures. J Chem Thermodyn 135:241-251. https://doi.org/10.1016/j.jct.2019.03.014

Ferreira AM, Esteves PDO, Boal-Palheiros I, Pereiro AB, Rebelo LPN, Freire MG (2016) Enhanced tunability afforded by aqueous biphasic systems formed by fluorinated ionic liquids and carbohydrates. Green Chem 18(4):1070-1079. https://doi.org/10.1039/C5GC01610J

Freire MG, Louros CLS, Rebelo LPN, Coutinho JAP (2011) Aqueous biphasic systems composed of a water-stable ionic liquid + carbohydrates and their applications. Green Chem 13(6):1536-1545. https://doi.org/10.1039/ C1GC15110J

Hadzir MH, Abbasiliasi S, Ariff AB, Yusoff SB, Ng HS, Tan JS (2016) Partitioning behavior of recombinant lipase in Escherichia coli by ionic liquid-based aqueous two-phase systems. RSC Adv 6(86):82571-82580. https://doi. org/10.1039/C6RA16722E

Johansson H-O, Feitosa E, Junior AP (2011) Phase diagrams of the aqueous two-phase systems of poly(ethylene glycol)/sodium polyacrylate/salts. Polymers 3(1):587-601

Miyawaki O, Tatsuno M (2011) Thermodynamic analysis of alcohol effect on thermal stability of proteins. J Biosci Bioeng 111(2):198-203. https://doi. org/10.1016/j.jbiosc.2010.09.007

Neves CMSS, Sousa RdCS, Pereira MM, Freire MG, Coutinho JAP (2019) Understanding the effect of ionic liquids as adjuvants in the partition of biomolecules in aqueous two-phase systems formed by polymers and weak salting-out agents. Biochem Eng J 141:239-246. https://doi. org/10.1016/j.bej.2018.10.022

Ng HS, Tan CP, Chen SK, Mokhtar MN, Ariff A, Ling TC (2011) Primary capture of cyclodextrin glycosyltransferase derived from Bacillus cereus by aqueous two phase system. Sep Purif Technol 81(3):318-324. https://doi. org/10.1016/j.seppur.2011.07.039

Ng HS, Tan CP, Mokhtar MN, Ibrahim S, Ariff A, Ooi CW, Ling TC (2012) Recovery of Bacillus cereus cyclodextrin glycosyltransferase and recycling of phase components in an aqueous two-phase system using thermo-separating polymer. Sep PurifTechnol 89:9-15. https://doi.org/10.1016/j.seppu r.2011.12.028

Ng HS, Ooi CW, Show PL, Tan CP, Ariff A, Moktar MN, Ng E-P, Ling TC (2014) Recovery of Bacillus cereus cyclodextrin glycosyltransferase using ionic liquid-based aqueous two-phase system. Sep Purif Technol 138:28-33. https://doi.org/10.1016/j.seppur.2014.09.038

Ng HS, Chai CXY, Chow YH, Loh WLC, Yim HS, Tan JS, Lan JC (2018) Direct recovery of Bacillus subtilis xylanase from fermentation broth with an 
alcohol/salt aqueous biphasic system. J Biosci Bioeng 125(5):585-589. https://doi.org/10.1016/j.jbiosc.2017.12.010

Ng H-S, Ng T-C, Kee PE, Tan JS, Yim HS, Lan JC-W (2019) Partition efficiency of cytochrome $c$ with alcohol/salt aqueous biphasic flotation system. J Biosci Bioeng 129(2):237-241. https://doi.org/10.1016/j.jbiosc.2019.08.013

Ooi CW, Tey BT, Hii SL, Kamal SMM, Lan JCW, Ariff A, Ling TC (2009) Purification of lipase derived from Burkholderia pseudomallei with alcohol/salt-based aqueous two-phase systems. Process Biochem 44(10):1083-1087. https:// doi.org/10.1016/j.procbio.2009.05.008

Pei Y, Wang J, Wu K, Xuan X, Lu X (2009) lonic liquid-based aqueous two-phase extraction of selected proteins. Sep Purif Technol 64(3):288-295. https:// doi.org/10.1016/j.seppur.2008.10.010

Pereira MM, Pedro SN, Quental MV, Lima ÁS, Coutinho JAP, Freire MG (2015) Enhanced extraction of bovine serum albumin with aqueous biphasic systems of phosphonium- and ammonium-based ionic liquids. J Biotechnol 206:17-25. https://doi.org/10.1016/j.jbiotec.2015.03.028

Quental MV, Pereira MM, Ferreira AM, Pedro SN, Shahriari S, Mohamadou A, Coutinho JAP, Freire MG (2018) Enhanced separation performance of aqueous biphasic systems formed by carbohydrates and tetraalkylphosphonium- or tetraalkylammonium-based ionic liquids. Green Chem 20(13):2978-2983. https://doi.org/10.1039/C8GC00622A
Ran L, Yang C, Xu M, Yi Z, Ren D, Yi L (2019) Enhanced aqueous two-phase extraction of proanthocyanidins from grape seeds by using ionic liquids as adjuvants. Sep Purif Technol 226:154-161. https://doi.org/10.1016/j. seppur.2019.05.089

Sadeghi R, Ebrahimi N, Tehrani MD (2016) Investigation of carbohydrates as non-charged, non-toxic and renewable soluting-out agent for polymer based aqueous biphasic systems implementation. Polymers 98:365-377. https://doi.org/10.1016/j.polymer.2016.06.050

Wan PK, Lan JC-W, Chen P-W, Tan JS, Ng HS (2018) Recovery of intracellular ectoine from Halomonas salina cells with poly(propylene) glycol/salt aqueous biphasic system. J Taiwan Inst Chem Eng 82:28-32. https://doi. org/10.1016/j.jtice.2017.11.003

Wang Y, Han J, Xu X, Hu S, Yan Y (2010) Partition behavior and partition mechanism of antibiotics in ethanol/2-propanol-ammonium sulfate aqueous two-phase systems. Sep Purif Technol 75(3):352-357. https://doi. org/10.1016/j.seppur.2010.09.004

\section{Publisher's Note}

Springer Nature remains neutral with regard to jurisdictional claims in published maps and institutional affiliations.

\section{Submit your manuscript to a SpringerOpen ${ }^{\odot}$ journal and benefit from:}

- Convenient online submission

- Rigorous peer review

- Open access: articles freely available online

- High visibility within the field

- Retaining the copyright to your article

Submit your next manuscript at $\boldsymbol{\text { springeropen.com }}$ 